\title{
Effects of molecular dipole orientation on the exciton binding energy of $\mathrm{CH}_{3} \mathrm{NH}_{3} \mathrm{PbI}_{3}$
}

\author{
Carlo Motta, ${ }^{1, *}$ Pankaj Mandal, ${ }^{1,2}$ and Stefano Sanvito ${ }^{1}$ \\ ${ }^{1}$ School of Physics, AMBER and CRANN Institute, Trinity College, Dublin 2, Ireland \\ ${ }^{2}$ Indian Institute of Technology Bombay, Powai, Mumbai 400076, India
}

(Received 4 April 2016; published 5 July 2016)

\begin{abstract}
We present a simple interacting tight-binding model for excitons, which is used to investigate the dependence of the exciton binding energy of $\mathrm{CH}_{3} \mathrm{NH}_{3} \mathrm{PbI}_{3}$ over the disorder induced by the molecular motion at room temperature. The model is fitted to the electronic structure of $\mathrm{CH}_{3} \mathrm{NH}_{3} \mathrm{PbI}_{3}$ by using data from density-functional theory and Born-Oppenheimer ab initio molecular dynamics, and it is solved in the mean-field approximation. When a finite-scale analysis is performed to extract the energetic of the excitons at experimental concentrations we find that disorder in general reduces the binding energy of about $10 \%$. This suggests that the excitonic properties of $\mathrm{CH}_{3} \mathrm{NH}_{3} \mathrm{PbI}_{3}$ largely depend on the electronic structure of the $\mathrm{PbI}_{3}$ inorganic lattice.
\end{abstract}

DOI: 10.1103/PhysRevB.94.045202

\section{INTRODUCTION}

The understanding of the electronic processes in many materials often requires a careful analysis of the disorder intrinsically present. This is particularly true in the case of organic compounds. For instance, static disorder is omnipresent in polymers due to their large polymorphism, while in small-molecule organic crystals large thermal fluctuations, caused by the weak dispersive bonds between the molecules, leads to dynamical disorder at any practical temperature. In both cases, the effect of the disorder is to determine the electron transport properties [1]. When a material is hybrid, namely it is composed of an organic and an inorganic component, some of these characteristics remain [2,3].

Hybrid perovskites are a class of compounds with the general chemical formula $A B X_{3}$, where $A$ is an organic cation located in an inorganic cage of corner-sharing octahedra constituted by a central cation, $B(B=\mathrm{Pb}, \mathrm{Sn})$, and six halide anions, $X(X=\mathrm{Cl}, \mathrm{Br}, \mathrm{I})$. A notable example of such family is $\mathrm{CH}_{3} \mathrm{NH}_{3} \mathrm{PbI}_{3}$, a material which has attracted huge recent interest as a light absorber in solar cell technology [4]. Such a class of compounds combines properties coming from two worlds, the organic and the inorganic, resulting from the intercalation of the two types of lattices. In fact, they possess excellent light absorption, large carrier diffusion length and mobility, and low defect activity, all properties typical of inorganic conventional semiconductors [5,6]. At the same time, they also show properties characteristic of organic materials such as easy molecular movement [7], a large dielectric constant, the presence of local dipole moments in the lattice [8], and long-range van der Walls interactions giving rise to soft low-energy phonon modes [9].

The organic cations in hybrid perovskite have been shown to display strong rotational disorder at room temperature, such that in all respects the system loses translational symmetry. The most realistic picture of this material is thus that of a disordered lattice, where the molecules are randomly oriented, and the typical reorientation time scale is of the order of picoseconds. This has been confirmed by $a b$ initio molecular dynamics $[10,11]$, by interatomic model potential simulations [12],

*mottac@tcd.ie and by direct vibrational anisotropy spectroscopy [13]. Such disorder is responsible for local variations of the electronic structure, with a critical impact in the optical region. In fact, the band gap, which electronically has to be attributed to the inorganic sublattice, is demonstrated to vary by up to $0.2 \mathrm{eV}$ depending on the orientation of the molecules $[10,14]$. Moreover, the nature of band gap itself is subject to variations, as it dynamically shifts from direct to indirect depending on the molecule position [15-17].

Upon illumination at the absorption edge, one finds a strong excitonic peak, which broadens with increasing temperature [18]. Such peak broadening has been attributed to the motion of the organic cations [18]. The magnitude of the exciton binding energy, $E_{B}$, has been debated recently. Most of the measurements report a room-temperature value in the 10 - to $30-\mathrm{meV}$ range [18], while a recent study shows that $E_{B}$ is $16 \mathrm{meV}$ at $4 \mathrm{~K}$ and rapidly collapses to few meV [19] as temperature increases to $300 \mathrm{~K}$. This temperature dependence suggests that the molecular motion has some impact on the exciton lifetime. The collective orientational motion of the organic cations has been proposed to screen the excitons and to favor their dissociation into free carriers [20], localizing electrons and holes in spatially distinct locations and thus reducing their recombination rate [11,21]. Hence, configurational disorder is proposed to contribute to the dissociation of the exciton by lowering the binding energy to generate free carriers at room temperature.

In order to capture the effect of disorder on the electronic properties of solids, one requires simulations on a large scale, such that many molecular units are included in the simulation cell. This is prohibitively expensive to $a b$ initio methods, because of the large computational overheads. Notably, one needs both large cells, able to describe the excitons formation at realistic concentrations, and extensive configuration averages over the disorder configurations. In order to achieve such a goal, in this work we propose a tight-binding (TB) model for excitons, which, despite its its simplicity, is able to capture the main physics of the hybrid perovskites. We demonstrate that the inclusion of disorder in general reduces the exciton binding energy. However, when the model is fitted to the electronic structure of $\mathrm{CH}_{3} \mathrm{NH}_{3} \mathrm{PbI}_{3}$ and solved for realistic exciton concentrations, we find that such a reduction is of the 
order of $10 \%$, i.e., that the excitonic properties are largely determined by the inorganic lattice alone.

The paper is organized as follows. After presenting the model and providing some general insights into the general features that it describes, we discuss the effect of dimensionality. Then we fit the TB parameters with the ab initio band structure of methylammonium lead iodide and investigate the effect of disorder on the exciton binding energy. Finally, we conclude.

\section{MODEL AND SOLUTION METHOD}

We construct a model of interacting electrons and holes based on the following tight-binding Hamiltonian [22,23]:

$$
\hat{H}=\hat{H}^{e}+\hat{H}^{h}+\hat{U}^{\text {int }} \text {, }
$$

where $\hat{H}^{\alpha}$ is the single-particle Hamiltonian for electrons $(\alpha=e)$ and holes $(\alpha=h)$,

$$
\hat{H}^{\alpha}=\sum_{i} \varepsilon_{0}^{\alpha} \hat{n}_{i}^{\alpha}+\sum_{i j} t_{i j}^{\alpha} \hat{a}_{i}^{\alpha \dagger} \hat{a}_{j}^{\alpha},
$$

and $\hat{U}^{\text {int }}$ is the interaction Hubbard-like term

$$
\hat{U}^{\mathrm{int}}=\sum_{i j} U_{i j} \hat{n}_{i}^{e} \hat{n}_{j}^{h} .
$$

Here $\hat{a}_{i}^{\alpha \dagger}\left(\hat{a}_{i}^{\alpha}\right)$ is the cre ation (annihilator) operator for the the quasiparticle $\alpha$ at the $i$ th site, $\hat{n}_{i}^{\alpha}=\hat{a}_{i}^{\alpha \dagger} \hat{a}_{i}^{\alpha}$ is the density operator, $\varepsilon_{0}^{\alpha}$ is the on-site energy, and $t_{i j}^{\alpha}$ the hopping integral. The attractive Coulomb interaction between the quasiparticle is long ranged and defined as

$$
U_{i j}=\left\{\begin{array}{ll}
-U_{0} & i=j \\
-\beta U_{0} / r_{i j} & i \neq j
\end{array},\right.
$$

where we keep $\beta=0.75$.

At this level, our model is identical to the one introduced in Ref. [23], where it was solved by exact diagonalization. In our case exact diagonalization, or another many-body approach, is not an option since we have to solve the model for large cells and consider a significant number of disorder realizations (see later). As a consequence, we propose a solution based on the mean-field approximation, which effectively transforms the problem into a single-particle one, although this requires the solution to be determined self-consistently. To this end, the density operator is expanded about the local quasiparticle density, $\hat{n}=\langle n\rangle+\delta n$, and neglect the fluctuationfluctuation term. The resulting mean-field Hamiltonian then reads

$$
\begin{aligned}
\hat{H}= & \sum_{i}\left(\varepsilon_{0}^{e}+\sum_{j} U_{i j}\langle n\rangle_{j}^{h}\right) \hat{n}_{i}^{e}+\sum_{i}\left(\varepsilon_{0}^{h}+\sum_{j} U_{i j}\langle n\rangle_{j}^{e}\right) \hat{n}_{i}^{h} \\
& +\sum_{i j}\left(t_{i j}^{e} \hat{a}_{i}^{e \dagger}+t_{i j}^{h} \hat{a}_{i}^{h \dagger}\right)-\sum_{i j} U_{i j}\langle n\rangle_{i}^{e}\langle n\rangle_{j}^{h}
\end{aligned}
$$

where the effect of the interaction is to renormalize the onsite energies of the quasiparticles. In the absence of Coulomb interaction the exciton energy is obtained as the difference between the lowest eigenvalues of the electrons system and the highest eigenvalue of the holes one, $E_{\text {tot }}\left(U_{0}=0\right)=\epsilon_{\min }^{e}-$ $\epsilon_{\max }^{h}$. In contrast, when the interaction is present, the exciton total energy can be written as $E_{\text {tot }}\left(U_{0} \neq 0\right)=\epsilon_{\min }^{e}-\epsilon_{\max }^{h}-$ $\sum_{i j} U\left(r_{i j}\right) n_{i}^{e} n_{j}^{h}$. Finally, the exciton binding energy is obtained as the difference $E_{B}=E_{\text {tot }}\left(U_{0}=0\right)-E_{\text {tot }}\left(U_{0} \neq 0\right)$.

In order to account for the disorder of real systems, we introduce in our model a random field. This is implemented by adding a small random fluctuation uniformly distributed in an interval $[-\xi, \xi]$ to the onsite potential at every site of the lattice. The disorder parameter $\xi$ has opposite sign for the electron and hole subsystems, meaning that an electron on-site disorder at site $i$ of $\xi_{i}$ corresponds to a hole on-site disorder of $-\xi_{i}$. This mimics the disorder introduced by a dipole moment. Calculations are then performed self-consistently for lattices of different size and for different disorder configurations.

Note that the orientation of the molecular cations in hybrid perovskites has two effects. On the one hand, it provides a local electric field originating from their permanent dipole. On the other hand, it generates distortions in the inorganic framework. As such, when the molecules are randomly oriented, the system is characterized by both a random dipolar and a distortion field, which translate into modifications of both the on-site and hopping parameters of our tight-binding model. This means that a more complete model should involve random fluctuations of both the on-site energy and the hopping integral, i.e., the model should include bond and on-site disorder. In general, the effects of these two types of disorder are equivalent, namely they promote charge localization, and the differences are only quantitative. Here, for simplicity, we consider only on-site disorder and we leave the investigation of both on-site and bond disorder for future work.

\section{RESULTS AND DISCUSSION}

\section{A. General features}

Let us start our analysis with presenting some general features of the model. In order to reduce the computational costs we begin with a simple cubic two-dimensional (2D) lattice and nearest neighbor interaction, which presents a density of state (DOS) with a single van Hove singularity at the band center. In the model the electron and hole systems have opposite on-site energies, $\varepsilon_{0}^{h}=-\varepsilon_{0}^{e}$, and identical hopping parameter. In Fig. 1(a) the DOS for the noninteracting system is plotted (gray area) for values of the parameters, $\varepsilon_{0}^{e}=5.5$ and $t_{i j}=1$, which return an exciton energy of $E_{\mathrm{tot}}=\epsilon_{\min }^{e}-$ $\epsilon_{\max }^{h}=2$. Note that for this first example we will report all quantities in units of the hopping integral, $t$. Note also that the DOS in Fig. 1 does not decay sharply at the band edges because of the artificial broadening introduced.

When the Coulomb interaction is switched on [see the dashed line in Fig. 1(a) for $U_{0}=3$ ] the two bands move closer to each other, since an attractive on-site potential moves upwards in energy-occupied bands and downwards in the empty ones. The net result is that the band gap and thus $\epsilon_{\min }^{e}-\epsilon_{\max }^{h}$ get reduced (for $U_{0}=3$ the computed value is $\epsilon_{\min }^{e}-\epsilon_{\max }^{h} \sim 0$ ) and the binding energy is no longer zero. In Fig. 1(b) we plot $E_{B}$ as a function of the interaction strength $U_{0}$, where a power law is found. This persists until $U_{0}=1$, beyond which there is a clear change in slope and the fit to the power law is less accurate. This is not surprising since for 

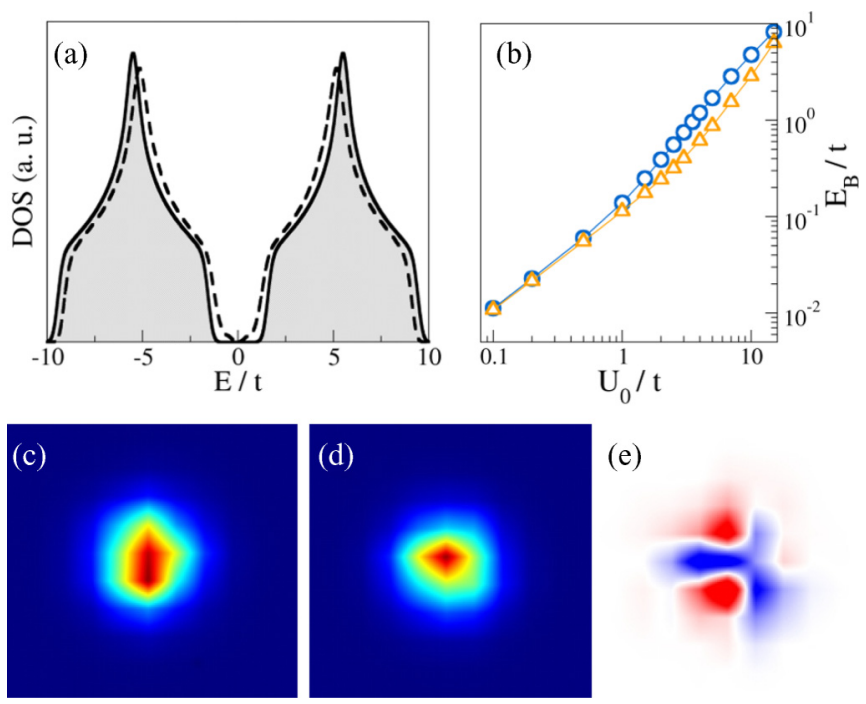

(e)

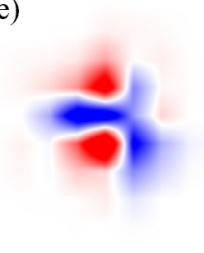

FIG. 1. General features of the model for a 2D square lattice with nearest-neighbor interaction: $\varepsilon_{0}^{h}=-\varepsilon_{0}^{e}=5 t$ and $t_{i j}=t$ (for a $20 \times 20$ lattice). In panel (a) we present the model DOS for the noninteracting case (gray area) and for $U_{0}=3$ (dashed line). Panel (b) shows the change of $E_{B}$ as a function of the interaction strength (on a log-log scale). Note the power-law dependence. The blue curve corresponds to a two-dimensional $30 \times 30$ lattice and the orange one to a three-dimensional $20 \times 20 \times 20$ lattice. In panels (c), (d), and (e) we plot the respectively $\langle n\rangle_{i}^{e},\langle n\rangle_{i}^{h}$, and $\Delta\langle n\rangle_{i}=\langle n\rangle_{i}^{e}-\langle n\rangle_{i}^{h}$, over a $20 \times 20$ grid. In panels (c) and (d) red indicates large charge density and blue small. In panel (e) blue is for a region of excess of hole density and red that of excess of electron density.

this disorder-free case one has $\langle n\rangle_{i}^{h}=-\langle n\rangle_{i}^{e}=1$, so the gap closes for $U_{0}=1$.

For a disorder-free lattice the electron and hole charge densities are identically distributed in space and they do maintain the lattice symmetry. In contrast, our correlated disorder localizes the charge density and effectively separate electrons from holes. This is displayed in Fig. 1(c) where we present $\langle n\rangle_{i}^{e}$ and $\langle n\rangle_{i}^{h}$ for the disorder case of $\xi=0.5 t$ and in Fig. 1(e) where we show $\Delta\langle n\rangle_{i}=\langle n\rangle_{i}^{e}-\langle n\rangle_{i}^{h}$. All densities are plotted over a $20 \times 20$ lattice for a specific disorder realization. Note from the figure that now the electrons and holes distributions differ with regions of electron excess and regions of hole excess. Such spatial separation is expected in general to reduce the exciton binding energy. This is analyzed next for both 2D and 3D lattices.

In Fig. 2 we present the relative binding energy reduction, $\delta E_{B} / E_{B 0}$, between the disordered and the disorder-free case, $E_{B 0}$. For this example we have considered simple cubic lattices in the nearest-neighbor approximation in both $2 \mathrm{D}$ and $3 \mathrm{D}$. The DOS for the 3D case has no van Hove singularities and a $\sqrt{E}$ dependence at the band edges. In order to determine to what extent the disorder magnitude $\xi$ affects $E_{B}$, we performed calculations for both the two- and three-dimensional lattices. In particular, we present results respectively for $20 \times 20$ and $12 \times 12 \times 12$ lattices and a Coulomb attraction parameter, $U_{0}$, ranging between $1 t$ and $4 t$. Since disorder is introduced stochastically over the lattice, every disorder realization is associated with a different exciton binding energy. For this

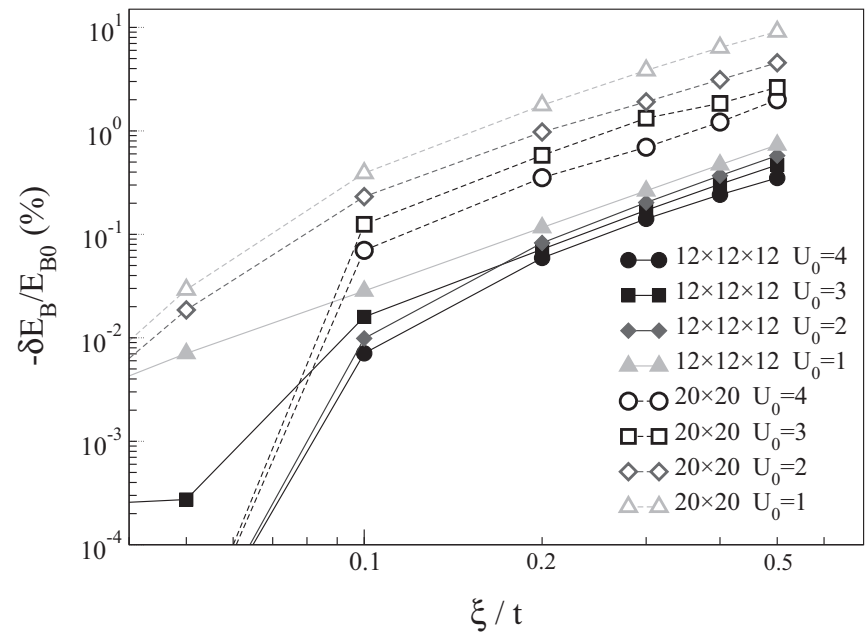

FIG. 2. Relative exciton binding energy reduction, $-\delta E_{B} / E_{B}$ as a function of the disorder strength, $\xi$, for two- and three-dimensional simple cubic lattices and calculated for different values of $U_{0}$ (note the log-log scale). The parameters used [in units of $t$ are $\varepsilon_{0}=5.5$ ( $2 \mathrm{D}$ case) and $\varepsilon_{0}=7$ ( $3 \mathrm{D}$ case) $]$. The error bars due to the disorder averages are of the same dimension of the symbols and are not shown.

reason each point in the graph is averaged over 20 independent disorder realizations.

In general, one can notice that $E_{B}$ decreases faster than a power law (note the log-log scale) as function of the disorder strength, regardless of the system dimensionality. Furthermore, there is a clear competition between disorder and interaction; for the same interaction strength, $\xi$, the reduction of the binding energy is less significant when $U_{0}$ is large for both the 2D and 3D cases. This is particularly dramatic at low disorder strength and large $U_{0}$. For instance, if one follows the $-\delta E_{B} / E_{B 0}(\xi)$ curve for $U_{0}=4$ it will be possible to note a change in slope at $\xi=0.1$. It is also interesting to note that the dimensionality of the system plays a role in the dependence of the binding energy with disorder, which is much more effective in the 2D case. For the choice of parameters used in Fig. 2 the largest $-\delta E_{B} / E_{B 0}(\xi)$ is about $10 \%$ for the $2 \mathrm{D}$ case (for $U_{0}=1$ and $\xi=0.5$ ), while it reaches only up to $1 \%$ for $3 \mathrm{D}$ lattices. This is expected as the screening in $2 \mathrm{D}$ is less efficient than in 3D, where the quasiparticle charge density has more freedom to respond to disorder. Finally, still in Fig. 2, we show $-\delta E_{B} / E_{B 0}(\xi)$ for the same $U_{0}=1$ but different lattice sizes, respectively $12 \times 12 \times 12$ and $20 \times 20 \times 20$. These effectively correspond to two different exciton densities (we have only one exciton per unit cell). We find that the exciton reduction is more pronounced for the lower density (larger lattice), as expected by the fact that a lower density implies a lower kinetic energy, and so a stronger sensitivity to disorder.

Note that there is a vast literature looking at the interplay between disorder and interaction, in particular related to the issue of metallicity in low dimensions and to the competition between Mott and Anderson insulators [24]. Here the scope of our work differs and we just aim at showing the effects of disorder on the binding energy of the excitons in perovskites. Furthermore, in our case the disorder is actually dynamic, with a typical timescale of picoseconds (the typical rotation time 
of the molecules). This essentially means that the interplay between the structure dynamics (phonons) and the charge carriers is the key factor determining charge transport in perovskites.

\section{B. Disorder in hybrid perovskites}

After having discussed the general trends of the model, we now adapt it to the case of the hybrid perovskites. First, we need to generate the relevant tight-binding parameters. We have then computed the band structure of cubic $\mathrm{CH}_{3} \mathrm{NH}_{3} \mathrm{PbI}_{3}$ by all-electron density-functional theory (DFT) including spinorbit coupling with the FHI-aims suite $[25,26]$. A mesh of $260 k$ points and the PBE functional [27] have been used, together with the "tight" basis set. The calculated valence and conduction bands are then fitted to a 2D simple cubic tight-binding model [see Fig. 3(a)], after the actual DFT band gap has been increased to the experimental one of $\sim 1.6 \mathrm{eV}$. The fit returns the following tight-binding parameters $t^{e}=0.2 \mathrm{eV}$, $t^{h}=0.14 \mathrm{eV}, \varepsilon_{0}^{e}=2 \mathrm{eV}$, and $\varepsilon_{0}^{h}=-1.7 \mathrm{eV}$, which will be used throughout.

The introduction of disorder in general produces a reduction of the band gap. This is shown for the noninteracting case in Fig. 3(b), where we plot the band gap as a function of $\xi$ with respect to that for $\xi=0, \Delta E_{\mathrm{GAP}}(\xi)=E_{\mathrm{GAP}}(\xi)-E_{\mathrm{GAP}}(\xi=0)$. We now need to provide a quantitative estimate of the disorder strength related to $\mathrm{CH}_{3} \mathrm{NH}_{3} \mathrm{PbI}_{3}$. To achieve this goal we have performed ab initio Born-Oppenheimer molecular dynamics (BOMD) for a $2 \times 2 \times 2$ cubic supercell of $\mathrm{CH}_{3} \mathrm{NH}_{3} \mathrm{PbI}_{3}$ at $300 \mathrm{~K}$. Here the $k$ mesh has been reduced to 14 points and the Bussi-Donadio-Parrinello thermostat [28] has been used. The inset of Fig. 3(b) shows the time-dependent band gap as calculated from the molecular dynamics trajectories after an initial equilibration period of $5 \mathrm{ps}$. We note that the fluctuations are of the order of $0.2 \mathrm{eV}$, in agreement with previous similar simulations $[10,11]$. When this results is compared with the $\Delta E_{\mathrm{GAP}}(\xi)$ curve of Fig. 3(b) we conclude that $\xi=0.4$ provides a good description of the disorder generated by the molecular motion at room temperature.
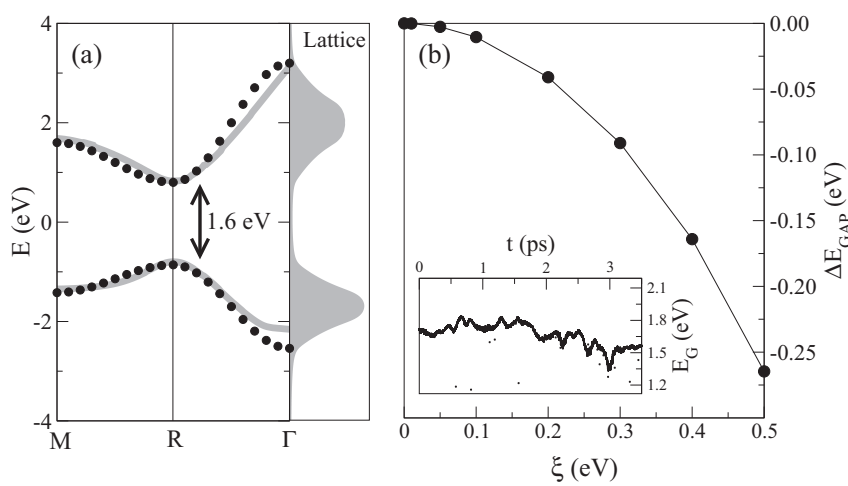

FIG. 3. The exciton model adapted to $\mathrm{CH}_{3} \mathrm{NH}_{3} \mathrm{PbI}_{3}$. (a) DFTcalculated conduction and valence bands (gray) and TB band structure (black dots) computed for a simple cubic lattice with parameters $t^{e}=0.2 \mathrm{eV}, t^{h}=0.14 \mathrm{eV}, \varepsilon_{0}^{e}=2 \mathrm{eV}$, and $\varepsilon_{0}^{h}=-1.7 \mathrm{eV}$. The TB DOS is plotted as a gray area on the right-hand side. (b) The bandgap variation is plotted as a function of $\xi$ for the same lattice. The inset shows the evolution of the band gap of $\mathrm{MAPbI}_{3}$ in a BOMD simulation.

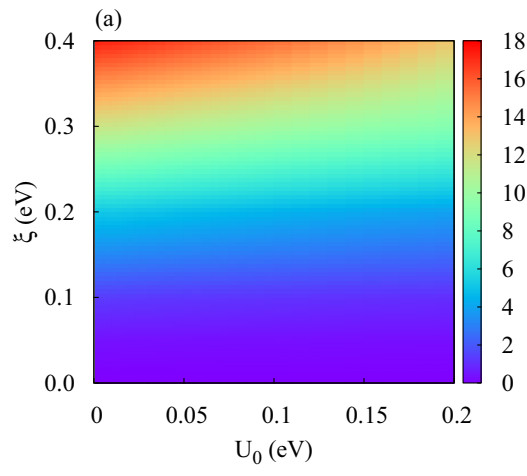

(b)

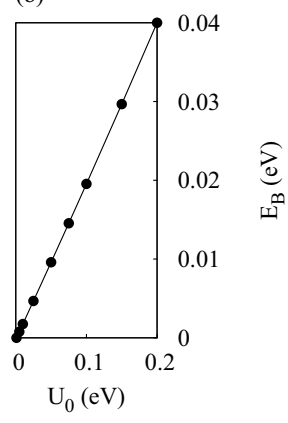

FIG. 4. (a) Exciton binding energy reduction $-\delta E_{B} / E_{B 0}$ as a function of the disorder and interaction strength for a 3D $12 \times 12 \times$ 12 lattice. The parameters used at those of $\mathrm{CH}_{3} \mathrm{NH}_{3} \mathrm{PbI}_{3}$, namely $t^{e}=0.2 \mathrm{eV}, t^{h}=0.14 \mathrm{eV}, \varepsilon_{0}^{e}=2 \mathrm{eV}$, and $\varepsilon_{0}^{h}=-1.7 \mathrm{eV}$. In panel (b) we show the exciton binding energy as a function of the Coulomb strength $U_{0}$ for $\xi=0$.

With this choice of TB parameters at hand we now discuss the dependence of the exciton binding energy on disorder. This is done at first with a $12 \times 12 \times 12$ cell in Fig. 4(a), where we show the exciton binding energy reduction, $-\delta E_{B} / E_{B 0}$, over a broad range of $U_{0}$ and $\xi$. The figure shows a significant reduction, up to $10-15 \%$, which is more marked in the top right corner of the plot where we find small $U_{0}$ and large $\xi$. Note that such a reduction is consistent with that reported in Fig. 2, since here we are looking at a parameter space region where $0<U_{0} / t<2$ and $0<\xi / t<2.5$, i.e., within a range of strong disorder and relatively week interaction. Figure 4(b) shows the absolute value of the exciton binding energy for different values of $U_{0}$ in the disorder-free case. Notably, with this choice of parameters the exciton binding energy remains rather small.

Finally, the last step of our analysis consists in estimating the exciton binding energy reduction at realistic exciton concentrations. This requires a final estimate of the interaction strength, $U_{0}$, and of the cell size (in our model the exciton density is $1 / \Omega$ with $\Omega$ being the unit cell volume). The typical concentrations of photoexcited charge carriers in hybrid perovskites $[29,30]$ do not exceed $10^{14} \mathrm{~cm}^{-3}$, a low value if compared to typical impurity concentrations in doped semiconductors. By assuming that the exciton concentration is of the same order of magnitude and from the knowledge of the methylammonium lead iodide unit cell volume $\left(\sim 260 \AA^{3}\right.$, see Refs. $\left.[14,17]\right)$, we find that a cubic lattice of size $\sim 350 \times 350 \times 350$ yields an exciton density in the typical experimental range (we have assumed that there is a cubic perovskite unit cell at each site of our simple cubic lattice).

Unfortunately, we cannot carry out calculations for such large cells (the dimension of the Hamiltonian is about 10000000 ), so we perform a finite-size scaling analysis. We point out here that in our model only one electron-hole pair is present in the lattice, and therefore the concentration is determined by the lattice size, and the exciton-exciton interaction is neglected. We compute $E_{B}$ for various $\xi$ and average over 150 disorder configurations for lattice sizes ranging from $5 \times 5 \times 5$ to $13 \times 13 \times 13$. The results are 

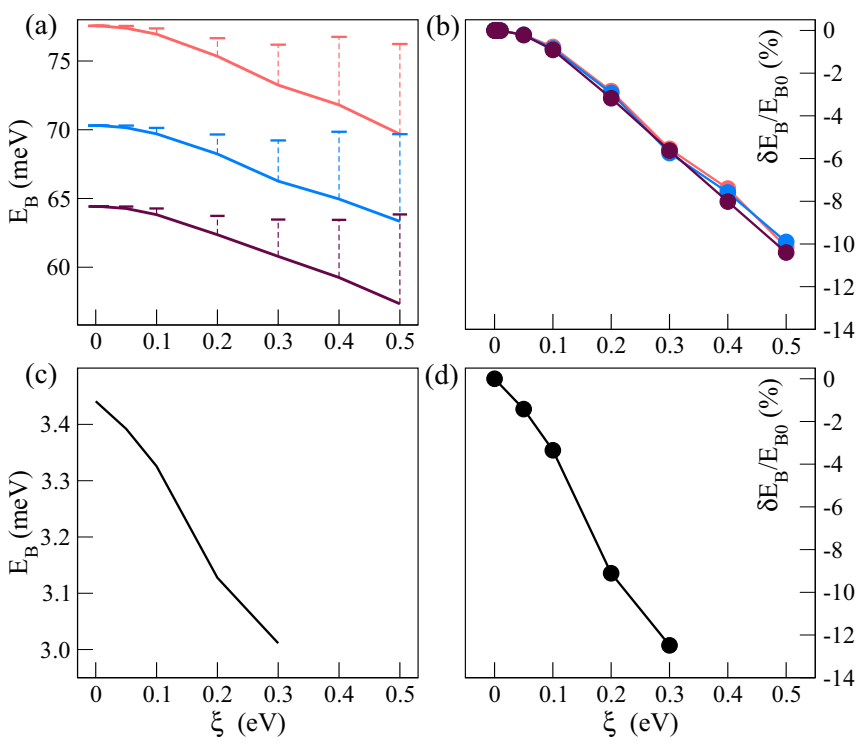

FIG. 5. Exciton binding energy as a function of disorder $\xi$ (a) and its percentage reduction (b) for exciton concentrations of $3 \times 10^{18}$ (red), $5 \times 10^{18}$ (blue), and $7 \times 10^{18}$ (maroon) $\mathrm{cm}^{-3}$. Statistics over 200 random configurations have been performed, and the standard deviation is shown in panel (a). The same quantities are shown in panels (c) and (d) for the experimental exciton concentration $\sim 10^{14} \mathrm{~cm}^{-3}$, as resulting from finite-size scaling.

then fitted to a power-law function and extrapolated to the $\sim 350 \times 350 \times 350$ lattice. Small variances of residuals in the range $10^{-1}-10^{-3}$ are found. The parameter $U_{0}$ is then set to a value $0.3 \mathrm{eV}$ providing an extrapolated binding energy of about $5 \mathrm{meV}$, which is in the experimental range at room temperature. The final result is displayed in Figs. 5(a) and 5(b), where we show the $E_{B}$ and its reduction as a function of $\xi$ for concentrations in the range of $10^{18} \mathrm{~cm}^{-3}$ and in
Figs. 5(c) and 5(d) the same quantities for a concentration of $10^{14} \mathrm{~cm}^{-3}$. Clearly, in all cases the reduction of $E_{B}$ is of the order of $10 \%$ for our realistic estimate of the disorder strength. We then conclude that in hybrid perovskites the exciton binding energy is indeed affected by disorder, but disorder can account for about $10 \%$ of the actual value of $E_{B}$. This essentially means that in hybrid perovskites $E_{B}$ is set by the details of the inorganic lattice, but its broadening is largely determined by the disorder in the molecular one.

\section{CONCLUSIONS}

In conclusion, we have constructed a simple exciton model based on a tight-binding Hamiltonian and Hubbard-like Coulomb interaction. The model can be solved for large disordered simulations cells in the mean-field approximation, providing insightful information about the dependence of the exciton binding energy on disorder. After having reviewed the general features of the model, we have adapted it to the case of $\mathrm{CH}_{3} \mathrm{NH}_{3} \mathrm{PbI}_{3}$, by fixing the parameters with DFT band structures, Born-Oppenheimer molecular dynamics, and by finite scaling analysis to realistic exciton concentrations. The model then suggests that disorder can account for fluctuations in the total exciton binding energy of the order of $10 \%$. This means that the exciton binding energy remains largely determined by the electronic structure of the inorganic $\mathrm{PbI}_{3}$ sublattice.

\section{ACKNOWLEDGMENTS}

This work is sponsored by the European Research Council QUEST project (Ref. No. 307891). Computational resources have been provided by the supercomputer facilities at the Trinity Center for High Performance Computing at ICHEC (Project No. tcphy066b). P.M. acknowledges additional financial support from CRANN during his stay in Dublin.
[1] D. P. McMahon and A. Troisi, Chem. Phys. Chem. 11, 2067 (2010).

[2] D. A. Egger, A. M. Rappe, and L. Kronik, Acc. Chem. Res. 49, 573 (2016).

[3] J. M. Frost and A. Walsh, Acc. Chem. Res. 49, 528 (2016).

[4] T. M. Brenner, D. A. Egger, L. Kronik, G. Hodes, and D. Cahen, Nat. Rev. Mater. 1, 15007 (2016).

[5] Y. Zhao and K. Zhu, Chem. Soc. Rev. 45, 655 (2016).

[6] S. Brittman, G. W. P. Adhyaksa, and E. C. Garnett, MRS Commun. 5, 7 (2015).

[7] A. M. A. Leguy, J. M. Frost, A. P. McMahon, V. G. Sakai, W. Kochelmann, C. Law, X. Li, F. Foglia, A. Walsh, B. C. O'Regan, J. Nelson, J. T. Cabral, and P. R. F. Barnes, Nat. Commun. 6, 7124 (2015).

[8] A. Stroppa, C. Quarti, F. D. Angelis, and S. Picozzi, J. Phys. Chem. Lett. 6, 2223 (2015).

[9] F. Brivio, J. M. Frost, J. M. Skelton, A. J. Jackson, O. J. Weber, M. T. Weller, A. R. Goñi, A. M. A. Leguy, P. R. F. Barnes, and A. Walsh, Phys. Rev. B 92, 144308 (2015).
[10] M. A. Carignano, A. Kachmar, and J. Hutter, J. Phys. Chem. C 119, 8991 (2015).

[11] C. Quarti, E. Mosconi, and F. De Angelis, Phys. Chem. Chem. Phys. 17, 9394 (2015).

[12] A. Mattoni, A. Filippetti, M. I. Saba, and P. Delugas, J. Phys. Chem. C 119, 17421 (2015).

[13] A. A. Bakulin, O. Selig, H. J. Bakker, Y. L. Rezus, C. Müller, T. Glaser, R. Lovrincic, Z. Sun, Z. Chen, A. Walsh, J. M. Frost, and T. L. C. Jansen, J. Phys. Chem. Lett. 6, 3663 (2015).

[14] C. Motta, F. El-Mellouhi, and S. Sanvito, Phys. Rev. B 93, 235412 (2016).

[15] C. Quarti, E. Mosconi, and F. D. Angelis, Chem. Mater. 26, 6557 (2014).

[16] A. Amat, E. Mosconi, E. Ronca, C. Quarti, P. Umari, M. K. Nazeeruddin, M. Grtzel, and F. D. Angelis, Nano Lett. 14, 3608 (2014).

[17] C. Motta, F. El Mellouhi, S. Kais, N. Tabet, F. Alharbi, and S. Sanvito, Nat. Commun. 6, 7026 (2015).

[18] M. A. Green, Y. Jiang, A. M. Soufiani, and A. W.-Y. Ho-Baillie, J. Phys. Chem. Lett. 6, 4774 (2015). 
[19] A. Miyata, A. Mitioglu, P. Plochocka, O. Portugall, J. T.-W. Wang, S. D. Stranks, H. J. Snaith, and R. J. Nicholas, Nat. Phys. 11, 582 (2015).

[20] J. Even, L. Pedesseau, and C. Katan, J. Phys. Chem. C 118, 11566 (2014).

[21] J. Ma and L.-W. Wang, Nano Lett. 15, 248 (2015).

[22] H. Ishii, T. Nakayama, and J. Inoue, Surf. Sci. 514, 206 (2002).

[23] H. Ishii, T. Nakayama, and J.-i. Inoue, Phys. Rev. B 69, 085325 (2004).

[24] D. C. Tsui, Rev. Mod. Phys. 71, 891 (1999).
[25] V. Blum, R. Gehrke, F. Hanke, P. Havu, V. Havu, X. Ren, K. Reuter, and M. Scheffler, Comput. Phys. Commun. 180, 2175 (2009).

[26] W. P. Huhn, M. Scheffler, and V. Blum (unpublished).

[27] J. P. Perdew, K. Burke, and M. Ernzerhof, Phys. Rev. Lett. 77, 3865 (1996).

[28] G. Bussi, D. Donadio, and M. Parrinello, J. Chem. Phys. 126, 014101 (2007).

[29] F. Hao, C. C. Stoumpos, D. H. Cao, R. P. H. Chang, and M. G. Kanatzidis, Nat. Photon. 8, 489 (2014).

[30] S. N. Rashkeev, F. El-Mellouhi, S. Kais, and F. H. Alharbi, Sci. Rep. 5, 11467 (2015). 